\title{
RICOCHET OF DEFORMING PROJECTILES FROM DEFORMING PLATES
}

\author{
J.A. ZUKAS and B. GASKILL \\ Computational Mechanics Consultants, Inc. P.O. Box 11314 Baltimore, MD 21239-0314, U.S.A.
}

(Received 21 August 1995; in revised form 6 November 1995)

\begin{abstract}
Summary-Ricochet means rebound of a striker from the impacted surface (or penetration into a medium along a curved trajectory emerging through the impacted surface with a residual velocity). Changes in direction, velocity and rotational motion of the penetrator are due to several mechanisms. These include release of stored elastic impact energy; influence of surfaces, material interfaces and impact deformations in the target on the magnitude and direction of the resisting force during impact; resistance to motion due to drag and friction. The subject is of interest due to the need to establish safety zones and to design containment structures to guard against failure of rapidly moving machine parts, to protect outer components of space vehicles from the energetic debris spray resulting from oblique hypervelocity impact and to reconstruct bullet trajectories in forensic engineering. This paper contrasts two-dimensional plane strain calculations of ricochet with fully threedimensional simulations performed with Apollo, a three-dimensional Lagrangian finite element code for impact and explosive loading problems set up exclusively on personal computers and workstations. While some useful information can be extracted from plane strain calculations regarding the early stages of impact, the use of two-dimensional calculations to simulate fully three-dimensional phenomena with long response times (up to the millisecond regime) results in gross overestimation of deflections and is inherently dangerous. Copyright (C) 1996 Published by Elsevier Science Ltd.
\end{abstract}

Keywords: ricochet, penetration, high obliquity impact, forensic engineering.

\section{BACKGROUND}

When a striker rebounds from the impacted surface of a target, or penetrates along a curved trajectory emerging through the impacted surface with a residual velocity, its behavior is defined as ricochet. Three major factors affect changes in velocity and direction associated with ricochet:

(a) impact pressures compress and deform striker and target. The subsequent recovery of the stored elastic energy results in motion changes.

(b) the characteristics of the target (surface properties and geometry; material properties; material interfaces) and its subsequent deformation after impact govern the direction and magnitude of the resisting force resultant which acts on the striker.

(c) resistance to motion due to drag and friction reduces velocities.

The component of the resisting force resultant which is aligned with the direction of motion slows the penetrator, which may be a "drag" force. The component of the resultant which acts normal to the direction of motion causes direction change and may be thought of as the "lift" force. If the resisting force resultant does not act on a line which intersects the center of gravity, the striker experiences rotating and bending moments [1-2].

Ricochet is of interest for a number of reasons. First, there is interest in the basic mechanics of ricochet for rigid and deformable media. Among these is the ingenious work of Johnson, Sengupta and Ghosh [3-4] who fired long rods against relatively thick plates, both of Plasticine (modeling clay), at obliquities ranging from 0 to $<75^{\circ}$ (obliquity is the impact angle measured from the normal to the plate surface). They were able to ascertain the mechanics of target cratering and define conditions leading to projectile breakup. Many of their observations have since been verified computationally and experimentally for metallic materials. Tate [5] derived an expression for the ricochet angle (also measured from the plate normal) for long rods from thick plates which agreed well with the observations of Johnson, Sengupta and Ghosh. An extensive experimental program involving ricochet of metallic spheres from metallic targets was undertaken by Backman and Finnegan [6]. Considerable data on ricochet from sand, clay, water and concrete can be found in the reports by Recht and his colleagues, cited above, as well as Hutchings [7], Birkhoff [8], Bushkovitch [9], Kemper and Jones [10], Johnson and Daneshi [11-12], Daneshi and Johnson [13] and many others. 
The safety of personnel and equipment threatened by ricocheting projectiles or fragments has been an ongoing concern. Typical of such studies are the works of Dunn and Dotson [14], Hayes et al. [15] and Reches [16].

Current interests in ricochet include the areas of hypervelocity impact and forensic engineering. Much of the work in hypervelocity impact involves normal incidence. However, most impacts in reality occur at obliquity. Many space structures in use or in the design phase have irregular outer surfaces or protrusions which are vulnerable to the debris clouds that can be generated by the breakup of projectiles impinging at high obliquity. Schonberg and Taylor [17-18] and Schonberg [19] have experimentally investigated oblique hypervelocity impact and ricochet for aluminum dual-wall structures. They developed a set of empirical equations that characterize observed penetration phenomena as a function of the geometric and material properties of the impacted structure and the diameter, obliquity and velocity of the impacting projectile. Burke and Rowe [20] review bullet ricochet from the standpoint of forensics. A knowledge of the wounds suffered by shooting victims, the deformation of bullets or shotgun pellets and ricochet marks on surfaces at the scene of a crime can be instrumental in reconstruction of the shooting.

\section{NUMERICAL SIMULATION OF RICOCHET}

Ricochet is a fully three-dimensional phenomenon. There are pitifully few analytical models which treat oblique incidence. These tend to be either extremely simple or limited to the initial stages of the impact process. Not uncommonly, some empiricism is involved in model development. The ricochet problem can therefore be treated experimentally or with finite element or finite difference codes as a fully three-dimensional solution or with the plane strain approximation.

Experiments take some time to set up, but once materials have been fabricated and the experimental arrangement is in place, 1-4 shots daily may be obtained, depending on whether the experiments are performed at full scale or model scale. Experiments are costly. A typical cost for a single full-scale shot is upwards of $\$ 10,000$, depending on the complexity of the experimental arrangement, the degree of instrumentation and the data reduction required. Model scale tests in enclosed ranges can be done for about $\$ 2,000$ per shot. Note that these costs are per shot, nor per data point. Frequently, because of excessive projectile yaw, malfunction of instrumentation or other causes, several shots may be required to obtain a valid data point. Even then, the information extracted from a ballistic test is minimal from the point of view of an analyst - initial and final velocity and orientation of the projectile, residual projectile mass, target deformation and mass loss. Inevitably there is scatter in the data due to variations in material properties of nominally identical materials and uncertainties in initial and boundary conditions. Time and cost constraints almost never permit acquisition of a data base with enough variation of parameters to construct unambiguous analytical models.

The cost of three-dimensional simulations is also high, even on supercomputers [21]. The exact cost depends on the code and computer used, the spatial and temporal resolution, the number of sliding interfaces in the calculation and the constitutive model. However, the cost of a three-dimensional calculation on a supercomputer almost always exceeds the cost of an equivalent experiment. The advantage of the computations is the quantity of information obtained: full time-resolved displacements, strains, strain rates, momenta, energies, forces, moments and so on. Coupled with experimental data, this forms an excellent base for construction of approximate analytical models for parametric studies. The validity of computational results depends on the constitutive model employed, the source of data for the parameters of the constitutive model and the degree of material failure in the experiment and how successfully it is simulated computationally.

In order to avoid the high cost of computing in three dimensions, recourse is sometimes made to two-dimensional calculations employing the plane strain assumption. Two-dimensional plane strain calculations are straightforward enough, relatively inexpensive and provide some useful information about the early stages of impact. However, when the oblique impact of a striker is treated as the impact of an infinitely long wedge [see Fig. 1], important physical phenomena are being neglected, not the least of which are the out-of-plane motions leading to lateral stress relaxations. Useful qualitative information (and, if care is taken with the calculations, useful quantitative information) may be obtained from plane strain solutions for the early stages of an oblique impact. Their utility, however, degrades with increasing time after impact so that for late times, when important aspects of 

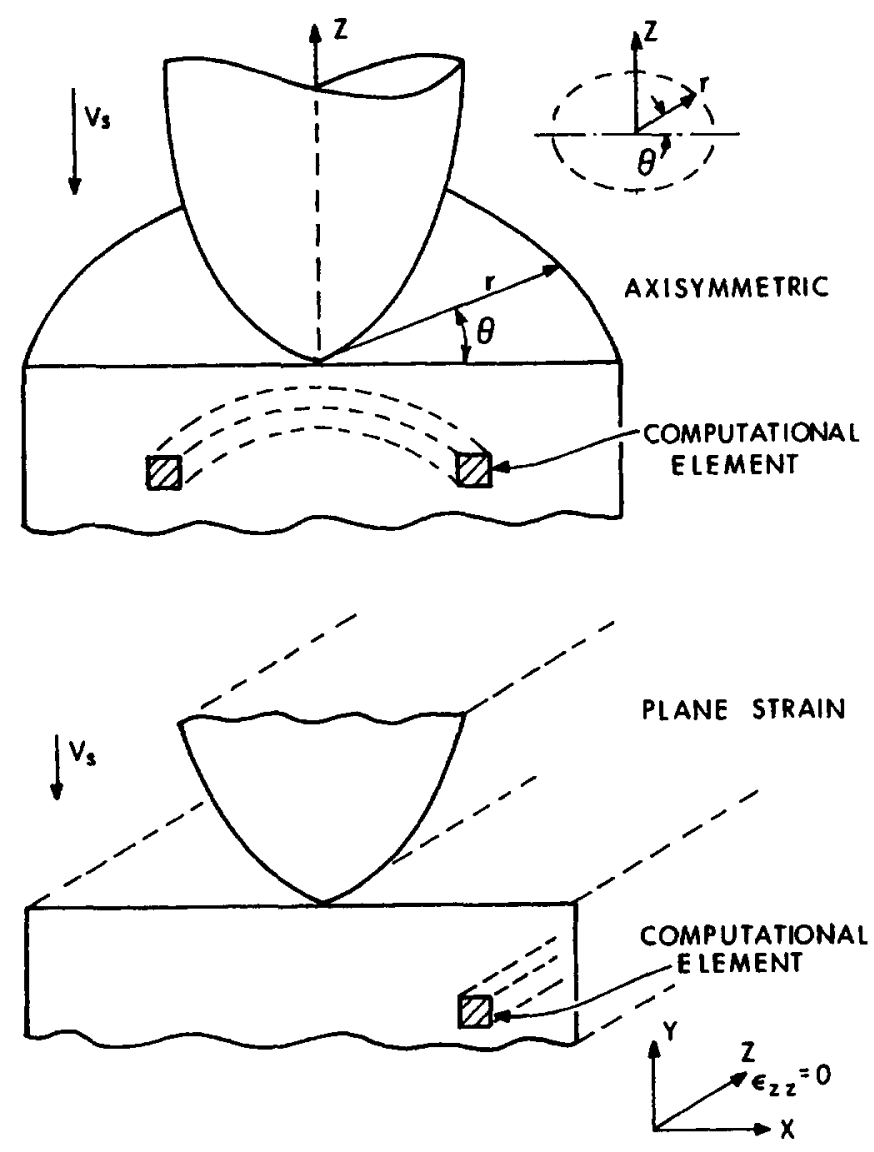

Fig. 1. Computational elements for axisymmetric and plane strain calculations.

penetration and target response (such as bending and shear failure) are being determined, plane strain solutions are speculative at best.

The nature of the plane strain approximation has been discussed by many authors [22-25]. The general conclusion of these studies is that, with appropriate scaling, useful information regarding overall kinetic energies, momenta and velocities may be obtained. However, after the time that a release wave would have returned from the lateral boundary of an actual three-dimensional calculation, plane strain calculations are not useful for extracting information related to the internal energy of the problem. They require less energy for deformation than their exact (2D axisymmetric or 3D oblique impact) counterparts and grossly overestimate late-time deformations.

If they are so fraught with risk, then, why perform plane strain calculations at all? Two reason stand out: they are cheap and, occasionally, they produce useful results. Norris et al. [26] used the HEMP code (existing production wave propagation codes are reviewed in detail in chapter 9 in Ref. [27]) in plane strain mode to supplement information from model scale oblique impact experiments of long rods striking thin plates at high obliquity and high velocity in order to determine optimum material and geometry configurations for high length-to-diameter $(L / D)$ ratio projectiles. They obtained excellent agreement between calculations and experiments, primarily because penetration was achieved at about the time the reflected wave from the penetrator's lateral dimension arrived. Zukas and Segletes [28] showed close correlation between experiments and calculations for hypervelocity projectiles striking spaced thin-plate targets at extreme obliquity for deformations and debris spray angles. Other simulations have been less successful. Jonas and Zukas [29] modeled long rod ricochet with the EPIC code. Good agreement between plane strain calculations and computed rod deformations was obtained early on after impact. However, comparison of calculations with radiographs at late times after impact showed poor correlation. The plane strain calculations severely underestimated rod bending. Detailed comparisons of the progressive deviation of plane strain calculations 
from exact (axisymmetric) calculations of long rod impact using the HELP code are shown by Zukas, Jonas and Misey [23].

Provided some care is taken in setting up calculations, and considerable skepticism employed in interpreting results, plane strain calculations can provide some insights into three-dimensional behavior. For highly energetic problems, the early-time response can be predicted quite well. However, it would be both foolish and dangerous to rely on plane strain calculations alone for design without corroboration by experiments or exact analyses.

\section{COMPUTATIONAL RESULTS}

\section{The ZeuS and Apollo codes}

Several calculations were performed to test the newly-developed Apollo code, a three-dimensional (3D) finite element Lagrangian code implemented on personal computers and workstations. The same calculations were performed in plane strain using ZeuS, a two-dimensional (2D) finite element code [30-37]. Results were compared with experiments performed by Ipson et al. [2].

Since the advent of hydrocodes in the early 1960s, a rich collection of algorithmic information has been accumulated for both Lagrangian and Eulerian calculations employing finite elements or finite differences. Hence it is much easier today to develop a computational tool than it was some 30 years ago when code developers were also pioneers. The development of Apollo made heavy use of existing algorithmic information, drawing very heavily on the work of Belytschko and his associates.

Apollo is an explicit time integration program for the nonlinear analysis of solids and fluids subjected to fast, transient loading. At present, the only element available is an isoparametric hexahedral element consisting of eight nodes, 24 degrees of freedom and six sides. A Lagrangian mesh description is employed, making use of Cauchy stresses and velocity strains together with the

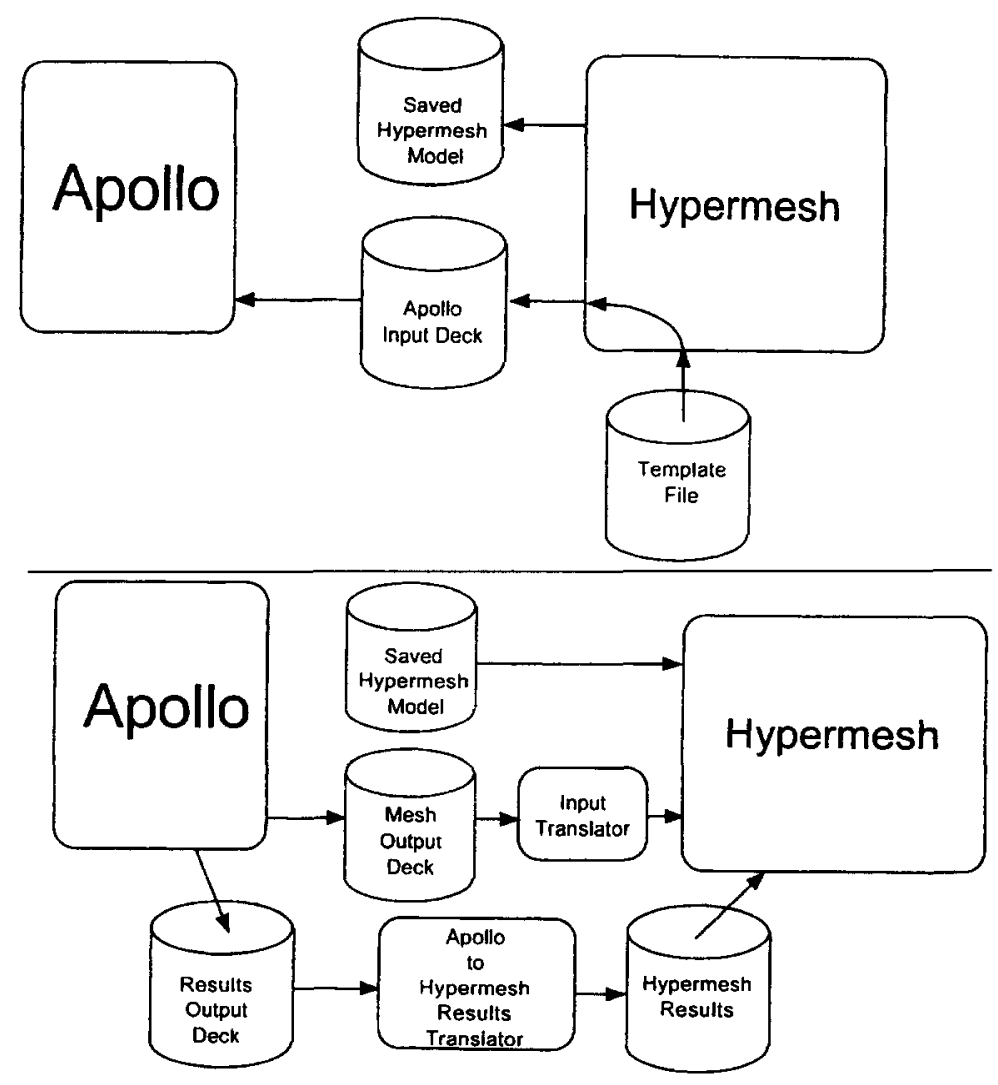

Fig. 2. Interaction between Apollo and Hypermesh. 
Table 1.

\begin{tabular}{lll}
\hline \multicolumn{3}{c}{ Material properties } \\
\hline & Projectile & Target \\
\cline { 2 - 3 } & 7.85 & 7.85 \\
Density (gm/cc) & $2.66 \mathrm{E}+07$ & $2.66 \mathrm{E}+07$ \\
Shear modulus (dynes/cm S*2) $\left.^{*}\right)$ & $2.00 \mathrm{E}+10$ & $1.14 \mathrm{E}+10$ \\
$\begin{array}{l}\text { Strength (dynes/cm**2) } \\
\text { (yield \& ultimate) }\end{array}$ & 1.2 & 1.6 \\
Strain at failure & & \\
\hline
\end{tabular}

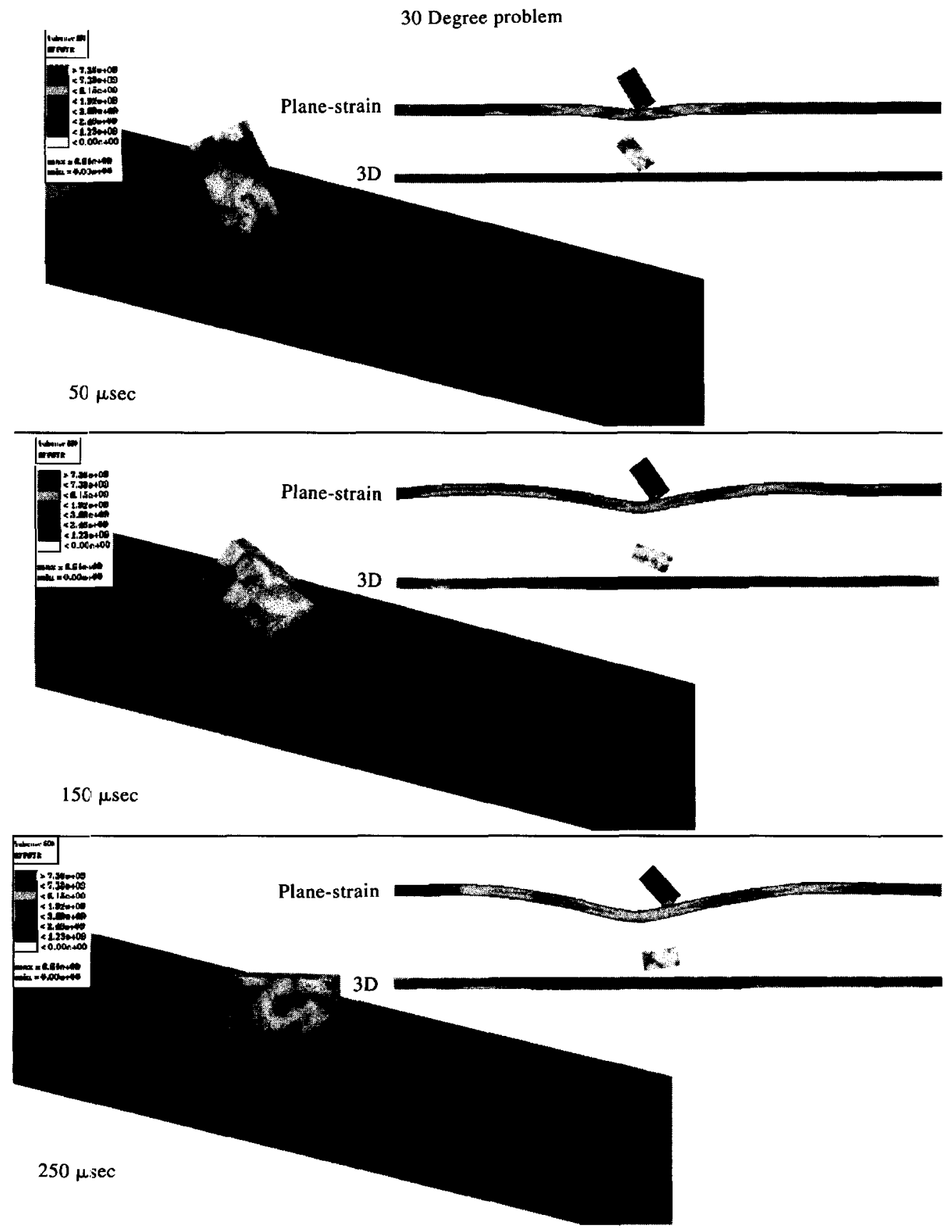

Fig. 3. Deformations at various times after impact $-30^{\circ}$ problem. 
Jaumann rotation rate. One quadrature point is used to evaluate stress and strain fields in the element. This implies that for evaluation of nodal forces, the stresses and strain rates are constant. The assumption of a constant stress field means that certain deformation modes of the element are not resisted by nodal forces. This phenomenon is known as hourglassing [38]. To avoid the severe mesh distortion brought about by hourglassing, a procedure developed by Flanagan and Belytschko [39] is used.

Apollo can support multiple materials in a single problem. Material strength is modeled with an elastic-viscoplastic hardening model. The von Mises yield criterion is used to detect the onset of plasticity. High-pressure response of materials is computed using the Mie-Gruneisen equation of state. An erosion criterion may be specified to remove excessively distorted elements from the calculation. When erosion occurs, new sliding surfaces are computed dynamically. This initial version of Apollo allows only a single sliding interface.

The Pinball algorithm [40] is used for contact-impact. The main concept of the Pinball algorithm is to enforce the impenetrability condition and contact conditions on a set of spheres, or pinballs,

30 Degree problem

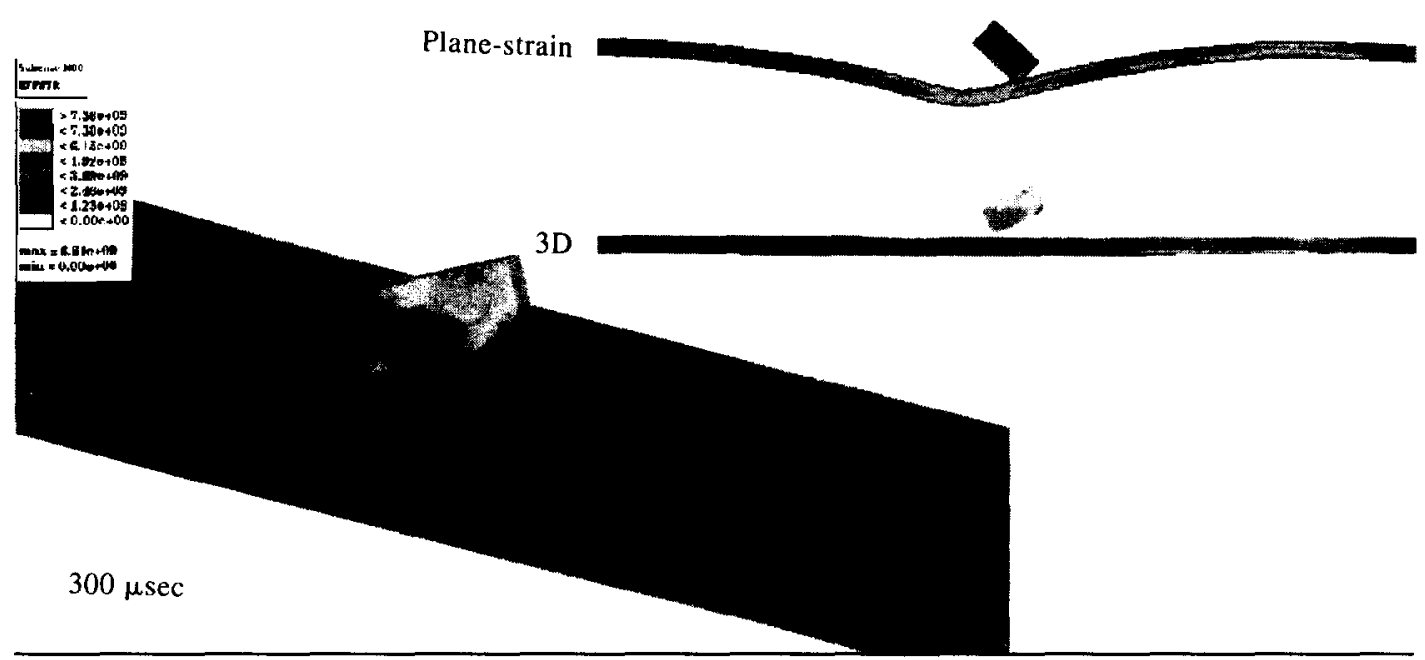

_. _ . . Experimental results (Recht and Ipson (1962))

Apollo 3D results

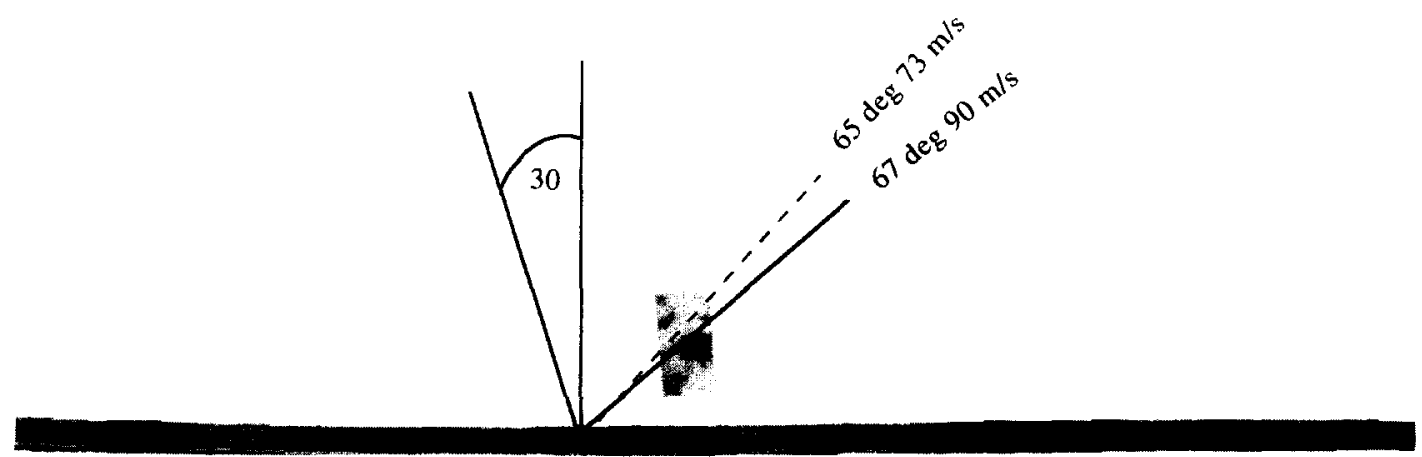

Fig. 4. Comparison of experimental and computational results. 
which are embedded in the finite elements. In the first cycle, the radius needed to create a sphere of volume equal to the element volume is computed for each element. For elastic-plastic problems most of the element deformations can be considered nearly incompressible. Therefore, the element volume, and also the radii of the pinballs, will change little over the course of a simulation. For this reason, the radii are calculated only once. For penetration detection on subsequent cycles, the distance between centers of each slave pinball and each master pinball are calculated and compared with the sum of the radii of the two elements. If the distance is less than that sum, penetration has occurred and a corrective force is applied. (See Refs [40-41] for details and applications).

The Pinball algorithm greatly reduces the time required in the search for penetration over conventional algorithms using put-back logic since interpenetrability becomes a simple check of distance between two pinballs and, since it involves almost no recursive calculations, it lends itself readily to vectorization. We have found that it works very well for impact calculations that are finely meshed in the vicinity of the contact point. If the element size is too large, if element aspect ratios greatly exceed one, or in low-speed impacts where the time of contact is crucial, then the pinball algorithm can be less than ideal. Because penetration by hexagonal elements is treated as penetration by spheres, penetration detection may be delayed at element corners and be premature at element faces. However, these details are insignificant compared to the time savings for high speed problems with apropriate meshes.

Pre- and post-processing for Apollo are done through the separate commercial package HyperMesh [42-44]. HyperMesh is a well-integrated finite element analysis (FEA) model design package that can be extremely flexible, depending on the application. It can serve as a post-processor as well. However, the underlying design of HyperMesh assumes that element connectivity will not change in the course of a calculation. This is true for Lagrangian calculations where deformations are limited. However, for large deformation problems experiencing material failure where element connectivity can change throughout the problem, many of the post-processing features of HyperMesh are lost. This applies to codes with erosion or element advection capabilities such as LS-DYNA, ZeuS, Apollo, PRONTO, DYSMAS-L, AUTODYN, EPIC and others under development.

A template file is read by HyperMesh and used to specify input-output characteristics between Apollo and HyperMesh [see Fig. 2]. The model is also saved in the HyperMesh native format. Apollo reads the resulting input deck generated by HyperMesh, performs the calculations to the specified problem time and creates a results output deck containing stresses, strains, pressures, velocities and other variables of interest. If connectivity changes occur in the course of a calculation a mesh output deck containing node and element information is generated as well. The results output deck is then translated into a results file native to HyperMesh. The mesh output information, if needed, can be loaded directly into HyperMesh by an input translator called by the HyperMesh application. Using

The 80 Degree problem

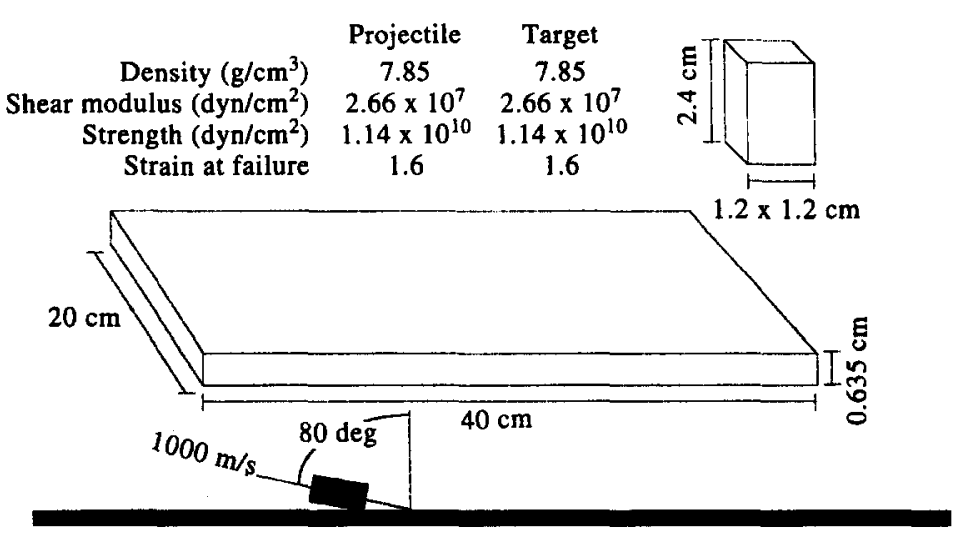

Fig. 5. Problem setup for $80^{\circ}$ impact. 
this informtion, the analyst can obtain from HyperMesh contour plotting, animation, displacement plots, cutaway views, rotations (depending on platform) and many other valuable aids to understanding the computational results.

\title{
2. Calculations
}

Several calculations were performed with ZeuS and Apollo to illustrate the differences between two-dimensional plane strain and fully three-dimensional calculations involving the oblique impact

\section{Degree obliquity}

\author{
Time $=5 \mu \mathrm{s}$
}

Plane-strain

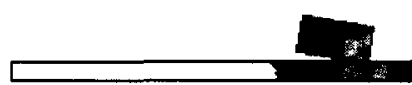

3D

\section{1}

Time $=25 \mu \mathrm{s}$

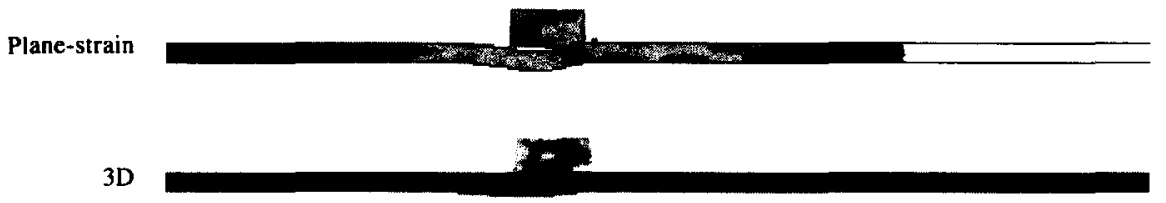

3D

Time $=75 \mu \mathrm{s}$
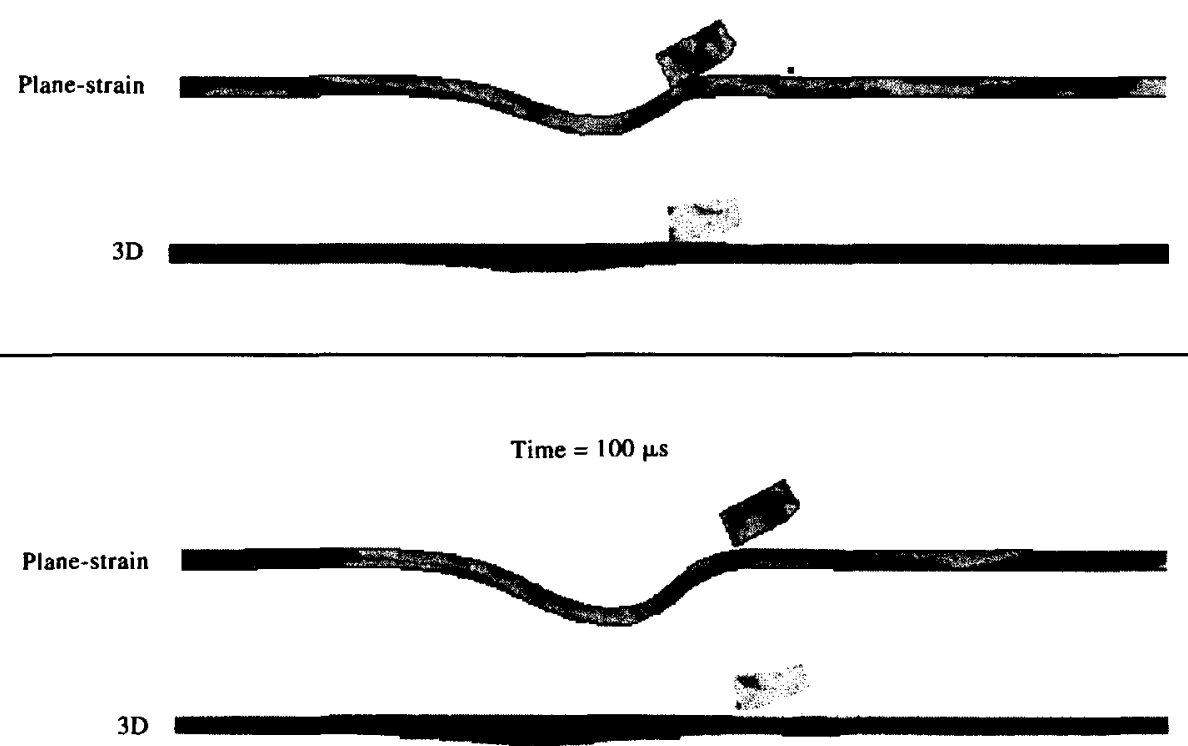

Fig. 6. Deformations at various times after impact $-80^{\circ}$ problem. 
and ricochet of compact projectiles. The first involved the impact of a hardened steel (Rockwell C 53) $1.27 \mathrm{~cm}$ rectangular cross-sectional projectile with $\mathrm{L} / \mathrm{D}=2$ and a mass of $26.5 \mathrm{~g}$ at an obliquity of $30^{\circ}$ and velocity of $180 \mathrm{~m} / \mathrm{s}$ against a 4130 steel plate with a thickness of $0.635 \mathrm{~cm}$. Material properties for the calculations are given in Table 1. Equation of state data for steel was taken from Ref. [45].

Figure 3 shows deformation profiles of the 2D and 3D calculations at three different times. At each time, an isometric view of the 3D calculation is presented which also shows surface stress contours. In addition, a cross-sectional view of the $2 \mathrm{D}$ plane strain calculation is shown and contrasted with an edge view of the $3 \mathrm{D}$ calculation. Note the progressive deviation of the $2 \mathrm{D}$ plane strain results from the 3D calculation with time. For this problem, the experimentally-determined ricochet angle was about $65^{\circ}$. (There is considerable scatter in the data [1].) The ricochet velocity was $73 \mathrm{~m} / \mathrm{s}$. The Apollo results indicate a ricochet angle of $67^{\circ}$ and a velocity of $90 \mathrm{~m} / \mathrm{s}$ (Fig. 4). No attempt was made to get similar information from the $2 \mathrm{D}$ calculation because of its obvious diversion from $3 \mathrm{D}$ results and reality.

Both calculations were run on a Silicon Graphics Indigo Extreme workstation. The 2D calculation employed 1,593 nodes and 2,736 elements and ran for a little over $68 \mathrm{~min}$, resulting in a whiz factor of $8.6 \times 10^{-5} \mathrm{CPU}$ seconds/node/cycle. The 3D computational grid consisted of 21,241 nodes and 15,432 elements. The total run time was $7.6 \mathrm{hr}$, giving a whiz factor of $1.3 \times 10^{-4} \mathrm{CPU}$ seconds/nodes/cycle.

A second set of calculations were performed using the same geometry and materials but changing the obliquity to $80^{\circ}$ and the striking velocity to $1 \mathrm{~km} / \mathrm{s}$. The initial configuration is shown in Fig. 5 . Computational results are shown in Fig. 6. Note that in this case, where the normal component of velocity is $174 \mathrm{~m} / \mathrm{s}$, less than one-fifth of the striking velocity, the 2D plane strain calculations still overestimate target deformation, which, in reality, for both cases was minimal, but otherwise mimics the three-dimensional results. The ricochet angle predicted from the $2 \mathrm{D}$ plane strain and $3 \mathrm{D}$ calculations was 68 and $72^{\circ}$, respectively. The same spatial resolution was used in the $80^{\circ}$ problem as in the $30^{\circ}$ problem. Total run time for the plane strain calculation was $110 \mathrm{~s}$ while the $3 \mathrm{D}$ calculation required $28 \mathrm{~min}$ to reach a problem time of $100 \mu \mathrm{s}$.

\section{CONCLUSIONS}

Plane strain calculations, if used with considerable caution, can produce results which have some qualitative value. However, because of the inherent difference in the physics modeled in the 2D plane strain and 3D calculations, good correlation between the two should not be expected. Plane strain results should never be used for design purposes unless corroborated by exact analyses, experiments or both.

\section{REFERENCES}

1. R. F. Recht and T. W. Ipson, The dynamics of terminal ballistics. Denver Research Institute, Final Report, Contract \# DA-23-072-ORD-1302, Denver, CO (AD 274128; appendix AD 328796) (1969).

2. T.W. Ipson, R. F. Recht and W. A. Schmeling, Effect of projectile nose shape on ballistic limit velocity, residual velocity and rocochet obliquity. Naval Weapons Center, Report NWC TP 5607, China Lake, CA (1973).

3. W. Johnson, A. K. Sengupta and S. K. Ghosh, High velocity oblique impact and ricochet mainly of long rod projectiles: an overview. Int. J. Mech. Sci., 24(7), 425-436 (1982).

4. W. Johnson, A. K. Sengupta and S. K. Ghosh, Plasticine modelled high velocity oblique impact and ricochet of long rods. Int. J. Mech. Sci., 24(7), 427-455 (1982).

5. A. Tate, A simple estimate of the minimum target obliquity required for the ricochet of a high speed long rod projectile. $J$. Phys. D: Appl. Phys., 12, 1825-1829(1979).

6. M. E. Backman and S. A. Finnegan, Dynamics of the oblique impact and ricochet of nondeforming spheres against thin plates. Naval Weapons Center, NWC TP 5844, China Lake, CA (1976).

7. I. M. Hutchings, The ricochet of spheres and cylinders from the surface of water. Int. J. Mech. Sci., 18, $243-247$ (1976).

8. G. Birkhof, Ricochet off land surfaces. Ballistic Research Laboratory, BRL Report 535, Aberdeen Proving Ground, MD (1945).

9. A. V. Bushkovitch, Ricochet of $0.30 \mathrm{cal}$. cylinders on striking an earth surface. Ballistic Research Laboratory, BRL Report 499, Aberdeen Proving Ground, MD (1944).

10. W. A. Kemper and J. C. Jones, Naval gunfire study - ricochet from water of $5 / 38^{\prime \prime}$ projectiles. Naval Weapons Laboratory, NWL TR 2252, Dahlgren, VA (1969).

11. W. Johnson and G. H. Daneshi, The trajectory of a projectile when fired parallel and near to the free surface of a plastic solid. Int. J. Mech. Sci., 20, 255-263, (1979).

12. W. Johnson and G. H. Daneshi, Results for the single ricochet of spherical-ended projectiles off sand and clay at up to $400 \mathrm{~m} / \mathrm{sec}$. In K. Kawata and J. Shioiri (eds), High Velocity Deformation of Solids, Springer-Verlag, Berlin (1979).

13. G. H. Daneshi and W. Johnson, Forces developed during the ricochet of projectiles of spherical and other shapes. Int. J. Mech. Sci., 19, 661-671 (1977). 
14. D. J. Dunn and W. D. Dotson, Jr., A method for estimating danger areas due to ricocheting projectiles. Ballistic Research Laboratory, BRL MR 1538, Aberdeen Proving Ground, MD (1964).

15. C. Hayes, W. Reeves and J. Collins, The effects of fragment ricochet on munition lethality. Air Force Armament Laboratory, AFATL-TR-75, 102, Eglin AFB, FL. (1975).

16. M. Reches, Fragment ricochet off homogeneous soils and its effects on weapons lethality. Army Material Systems Analysis Agency, AMSAA TM 79, Aberdeen Proving ground, MD (1970).

17. W. P. Schonberg and R. A. Taylor, Oblique hypervelocity impact response of dual-sheet structures, NASA-TM-100358 (1989).

18. W. P. Schonberg and R. A. Taylor, Penetration and ricochet phenomena in oblique hypervelocity impact. $A I A A, J ., 27(5)$ (1989).

19. W. P. Schonberg, Hypervelocity impact penetration phenomena in aluminum space structures. ASCE, J. Aerospace Engng., 3(3) (1990).

20. T. W. Burke and W. F. Rowe, Bullet ricochet: A comprehensive review, J. Forensic Sci., JFSCA 37(5), 1254-1260 (1992).

21. J. A. Zukas and K. D. Kimsey, Supercomputing and computational penetration mechanics. In R. F. Kulak and L. E. Schwer (eds), Computational Aspects of Contact, Impact and Penetration, Elmepress International, Lausanne (1991).

22. L. D. Bertholf, M. E. Kipp and T. W. Brown, Two-dimensional calculations for the oblique impact of kinetic energy projectiles with a multi-layered target. Ballistic Research Laboratory, BRL-CR-333, Aberdeen Proving Ground, MD (1977).

23. J. A. Zukas, G. H. Jonas and J. J. Misey, On the utility of the plane strain approximation for oblique impact computations. Ballistic Research Laboratory, BRL-MR-02969, Aberdeen Proving Ground, MD (AD-E430376) (1979).

24. T. W. Brown, Numerical modeling of oblique hypervelocity impact using two-dimensional plane strain models. In W. J. Nellis, L. Seaman and R. A. Graham (eds), Shock Waves in Condensed Matter-(1981), American Institute of Physics, NY (1982).

25. J. A. Zukas, Penetration and perforation of solids. Chapter 5 in J. A. Zukas, T. Nicholas, H. F. Swift, L. B. Greszczuk and D. R. Curran, Impact Dynamics, Wiley Interscience, NY (1982); republished by Krieger Publishing Co., Malabar, FL (1992).

26. D. M. Norris, J. K. Scudder, W. A. McMaster and M. L. Wilkins, Mechanics of long rod penetration at high obliquity. In Proc. High Density Alloy Penetrator Materials Conf., Army Materials and Mechanics Research Center, AMMRC-SP-77-3, Watertown, MA (1977).

27. J. A. Zukas (ed.), High Velocity Impact Dynamics. Wiley Interscience, NY (1990).

28. J. A. Zukas and S. B. Segletes, Hypervelocity impact on space structures. In T. L. Geers and Y. S. Shin (eds), Dynamic Response of Structures to High-Energy Excitations, AMD-Vol. 127/PVP-Vol. 225, American Society of Mechanical Engineers, NY (1991).

29. G. H. Jonas and J. A. Zukas, Mechanics of penetration: analysis and experiment. Int. J. Engng. Sci., 16, 879-903 (1978).

30. S. B. Segletes and J. A. Zukas, ZeuS user's manual; technical description and tutorial. Computational Mechanics Consultants, Inc., Baltimore, MD (1987).

31. S. B. Segletes and J. A. Zukas, The effect of material interfaces on calculations of plate penetration. In D. Hui and N. Jones (eds), Recent Advances in Impact Dynamics of Engineering Structures, AMD, Vol. 105, ASME, NY (1989).

32. S. B. Segletes and J. A. Zukas, Hypervelocity impact simulation of whipple shields. Proc. 17th Space Simulation Conf., Baltimore, MD, Nov 9-12 (1992).

33. S. B. Segletes and J. A. Zukas, The contact/erosion algorithm in the ZeuS hydrocode. In M. H. Aliabadi and C. A. Brebbia (eds), Contact Mechanics: Computational Techniques, Computational Mechanics Publications, Southampton, U.K. (1993).

34. B. Janzon, EFP modelling by numerical continuum dynamics on personal computers-a comparison between PCDYNA2D, ZeuS and AUTDYN-2D. Proc. 13 th Intl. Symp. on Ballistics, Stockholm, Sweden, June 1-3 (1992), American Defense Preparedness Association, Rosslyn, VA (1992).

35. J. A. Zukas, Some common problems in the numerical modeling of impact phenomena. Comp. Sys. Engng, 4(1), 43-58 (1993).

36. J. A. Zukas, Verification, validation and selection of wave propagation codes for penetration mechanics. DNA Conf. Verification and Validation of Nonlinear Structural Dynamics Codes, Washington, DC (1993).

37. J. A. Zukas and S. B. Segletes, Numerical modeling of hypervelocity impact phenomena with desktop computers. Adv. Engng Software, 14, 77-84 (1993).

38. T. Belytschko, Hourglass control in linear and nonlinear problems. Comp. Methods Appl. Mech. Engng, 43, 251-276(1984).

39. D. P. Flanagan and T. Belytschko, A uniform strain hexahedron and quadrilateral with orthogonal hourglass control, Int. J. Num. Methods Engng, 17, 679-706 (1981).

40. M. O. Neal, Contact-impact by the pinball algorithm with penalty, projection and augmented lagrangian methods. $\mathrm{PhD}$ Dissertation, Northwestern University, U.S.A. (1989).

41. T. Belytschko and M. O. Neal, The vectorized pinball contact impact routine. In A. H. Hadjian (ed.), Trans. 10th Int. Conf. on Structural Mechanics in Reactor Technology, Anaheim, CA (1989).

42. B. Corman, HyperMesh learning guide and tutorial. Altair Computing Inc., 1757 Maplelawn, Troy, MI 48084 (1992).

43. D. Hill, HyperMesh User's Manual, ibid (1994).

44. D. Hill, HyperMesh Programmer's Manual, ibid (1994).

45. B. J. Kohn, Compilation of hugoniot equations of state. Air Force Weapons Laboratory, AFWL-TR-69-38 (1969). 